\title{
Relay Deployment and Power Control for Lifetime Elongation in Sensor Networks
}

\author{
Yufeng Xin, Tuna Güven, Mark Shayman \\ Institute of Advanced Computer Studies \\ University of Maryland, College Park, MD 20742 \\ e-mail: $\{$ yxin, tguven, shayman\}eng.umd.edu
}

\begin{abstract}
In a sensor network, usually a large number of sensors transport data messages to a limited number of sinks. Due to this multipoint-to-point communications pattern in general homogeneous sensor networks, the closer a sensor to the sink, the quicker it will deplete its battery. This unbalanced energy depletion phenomenon has become the bottleneck problem to elongate the lifetime of sensor networks. In this paper, we consider the effects of joint relay node deployment and transmission power control on network lifetime. Contrary to the intuition the relay nodes considered are even simpler devices than the sensor nodes with limited capabilities. We show that the network lifetime can be extended significantly with the addition of relay nodes to the network. In addition, for the same expected network lifetime goal, the number of relay nodes required can be reduced by employing efficient transmission power control while leaving the network connectivity level unchanged. The solution suggests that it is sufficient to deploy relay nodes only with a specific probabilistic distribution rather than the specifying the exact places. Furthermore, the solution does not require any change on the protocols (such as routing) used in the network.
\end{abstract}

\section{INTRODUCTION}

Sensor nodes are low-cost, low power devices equipped with sensing, communication and processing capabilities albeit with finite and nonrenewable battery energy. Naturally, energy efficiency is a major concern in sensor networks. As communication in sensor networks is generally multipoint-topoint (i.e., from sensor nodes to an information sink node), sensor nodes in the proximity of the sink node have to relay comparably excessive traffic especially when the number of nodes in the network is large. For this reason, such nodes inevitably deplete their energy rapidly regardless of the routing algorithm utilized. This is the main energy bottleneck in a typical sensor network.

To this end, Luo et al. proposed to change the position of the information sink from time to time [9]. Since the sink mobility changes the set of sensor nodes around the sink over time, the traffic load, and consequently the energy depletion rate discrepancy between the sensors becomes smaller. However, sink mobility is rather a complex solution with the necessity of carefully selecting the mobility parameters such as the trajectory and speed of the sink node. It also assumes that the sink node has no energy limitations as mobility comes with

Partially supported by AFOSR under grant F496200210217 and NSF under grant CNS-0519554 the cost of significant energy consumption. Furthermore, the sink mobility needs to be accompanied by a specific, complex routing algorithm to forward data from sensors to the mobile sink node (See [9]).

In another line of work, strategic placement of sensors in linear networks was studied [8]. The necessary distance between neighboring sensors is obtained in order to achieve a specified lifetime. The basic observation is that nodes closer to the sink have shorter mutual distance. However, the geographical distribution of sensor networks is often decided by the specified sensory task in a certain area. Hence, strategic placement of sensors that is not complying with the sensory task naturally leads to a strictly suboptimal sensor node distribution. This may incur an unacceptably high cost since the density of sensor nodes would be excessively high (as well as redundant from the sensory task point of view) around the sink.

In this paper, we focus on the aforementioned energy bottleneck issue and consider deploying additional relay nodes in order to improve network lifetime of the multipoint-to-point dense sensor networks. Relay deployment is further coupled with power control (i.e., intelligent assignment of transmission ranges for both sensor and relay nodes). Contrary to the intuition, we model relay nodes as simple devices. Specifically, we assume that the relay nodes are even simpler than the sensor nodes in the sense that they have all the capabilities of a sensor node with the exception that they do not possess any sensing component. Essentially, the relay nodes extend the network lifetime by remedying the excessive traffic burden of a subset of sensor nodes. Note that this model differs from the traditional models in which relay nodes have superior communication and processing capabilities. Furthermore, we consider a flat network architecture as opposed to a hierarchical model, where sensors and relay nodes communicate with the information sink via possibly a multi-hop route that consists of several sensor and/or relay nodes.

Intuitively, relay node density should increase more and more with decreasing distances to the sink node(s) since we need more relays to share the increasing traffic (i.e., energy) load. On the other hand, with denser node distribution, one can presumably decrease the transmission ranges in the regions of higher node density without affecting the connectivity properties of the overall network (e.g., $k$-connectedness). Hence, as the node density increases with the addition of the relay nodes, the 
energy consumption of sensor nodes reduces not only because the traffic load that a node is required to forward decreases but also the energy consumption per transmission is reduced as well. Therefore, relay density and transmission range should be decided jointly in optimizing the network lifetime.

An obvious and maybe simpler alternative solution one may consider is to deploy sensor nodes with different levels of battery energy in such a way that the initial battery energy is higher for the nodes that are closer to the sink node. This way the energy drainage due to heavy traffic burden around the sink can be handled by the additional battery energy installed in sensor nodes. However, it is easy to see that for the same given total energy expenditure, the joint relay deployment and power control method promises a longer network lifetime compared to deploying sensors with heterogeneous battery energy levels. This is due to the fact that adjusting transmission ranges results in additional energy savings which cannot be realized otherwise. In addition, one should note that it is often not possible to equip sensors with extended battery life due to hardware constraints on the size of the batteries.

Another advantage of deploying additional relay nodes is that once relay nodes are carefully deployed, no further complex protocols, like mobility and energy-efficient routing protocols, are needed. Moreover, the solution does not specify the exact locations of relay nodes; rather it suggests that it is sufficient for relay nodes to follow a specific probability distribution. This can be beneficial for certain applications where the exact locations of the nodes deployed cannot be specified (e.g., military battlefield networks). Therefore, it is our belief that the proposed solution is a solid alternative for the aforementioned network lifetime elongation problem.

The rest of the paper is organized as follows. In Section II, we describe the network model and state the relay placement problem. The analytical solutions and numerical results regarding relay density and transmission power control are presented in Section III. In Section IV we describe the experimental setup used for evaluation of the proposed solution and present the simulation results. Section V concludes the paper.

\section{Network Model and Problem Statement}

Consider a wireless sensor network with static sensor nodes and one information sink that is responsible to collect all the data generated by the sensor nodes. Let $S=\{1, \ldots, S\}$ be the set of sensor nodes. Assume that the sensor node distribution follows a Poisson process with fixed density $\rho_{s}$ within a circle $C$ of radius $D$ and center $O$. The value of $\rho_{s}$ is in general decided by the specific sensory task under consideration. Each sensor node creates data traffic at a constant rate $\lambda$ and the sink node is located at the center $O$ of the circle $C$.

We consider deploying additional relay nodes within $C$ to prolong the network lifetime. Let $\mathcal{R}=\{1, \ldots, R\}$ be the set of relay nodes to be added. Relay node density should increase with decreasing distance $(0<d \leq D)$ to the sink node as mentioned in the previous section. Toward this end, we assume that relay nodes are distributed using a nonhomogeneous

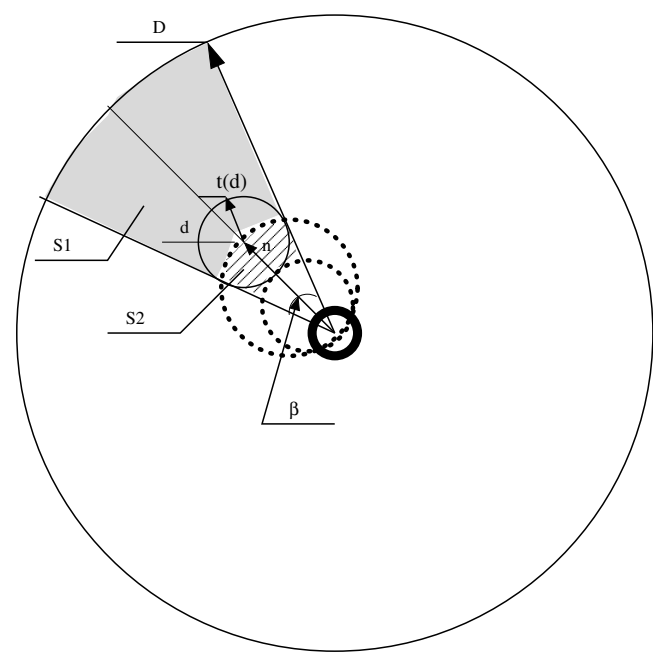

Fig. 1. Load distribution model $\left(d \geq t_{0}\right) . S_{1}$ and $S_{2}$ are two disjoint parts of a sector whose two sides are tangent to a circle with center $n$ and radius $t(d)$ [9].

Poisson process [10] with density $\rho_{r}(d)$ in contrast with the sensor nodes. Note that here we make use of the symmetry arising from the specific distribution of sensor nodes as well as the location of the sink node and define $\rho(d)$ as a function of $d$. Hence, the overall node distribution also follows a Poisson process with density $\rho(d)=\rho_{s}+\rho_{r}(d)$. Our objective is to find the optimal relay density $\rho_{r}(d)$ achieving maximum expected network lifetime when sensor nodes have a fixed and nonrenewable battery energy $B$.

We assume that all nodes (sensors, relays and the sink) use a common frequency channel. Let $t_{s} \ll D$ denote the maximum transmission range a node can use to transmit. As discussed earlier, nodes can presumably have shorter transmission ranges in the regions with a higher node density while keeping the network connectivity the same. It is a consequence of this fact to define the transmission ranges of nodes also as a function of distance $d$ to $O$; i.e., $t(d) \leq t_{s}$. In the following analysis we will assume that $t(d)=t_{0}$ for $d \leq t_{0}$. This simplifying assumption merely states that one-hop neighbors of the sink node use a common transmission range. Note that $t(d)$ is a nondecreasing function of $d$, i.e., $t_{0}$ represents the minimum transmission range used in the network. The value of $t_{0}$ is naturally a design parameter to be optimized.

We represent the original sensor network without the relays as $\mathbf{G}\left(t_{s}, \mathcal{S}\right)$ and we assume that $\rho_{s}$ is selected such that $\mathbf{G}\left(t_{s}, \mathcal{S}\right)$ is $k$-connected ${ }^{1}$ with a high probability $p_{0}$ (e.g., $p_{0}=0.999$ ), i.e.,

$$
\mathrm{P}\left(\mathbf{G}\left(t_{s}, \mathcal{S}\right) \in \mathbf{G}_{k}\right)=p_{0},
$$

where $\mathbf{G}_{k}$ is the set of $k$-connected graphs. Moreover, let $\mathbf{G}(t(d), \mathcal{S} \cup \mathcal{R})$ be the overall network graph obtained with the addition of relay nodes $\mathcal{R}=\{1, \ldots, R\}$ and by employing transmission power control.

\footnotetext{
${ }^{1} \mathrm{~A}$ graph is $k$-connected if and only if no set of $k-1$ nodes exists whose removal would disconnect the graph [4].
} 
As in [9], for simplicity we assume an ideal load-balanced shortest path routing (See [6] for a presentation of such protocols). We do not consider data aggregation. For simplicity, we define $\varepsilon(d)$ as the energy consumed to transmit a unit of data by a node at a distance $d$ from $O$ and assume that energy consumption for processing and receiving tasks is negligible. Specifically, we let $\varepsilon(d)=c(t(d))^{a}$, where $c$ is a constant and $a$ represents the attenuation exponent. It is typical to assume $2 \leq a \leq 4$.

We adopt the network lifetime definition presented in [2], which is the time span from the sensor deployment to the first loss of coverage. Specifically, we assume that each sensor has a limited sensing range. Let $C_{\rho_{s}}$ be the particular area in $C$ that is sensed by at least one sensor. Loss of coverage is defined as the time when some area within $C_{\rho_{s}}$ that is initially covered by the network is not sensed by any active node anymore.

A related system parameter is the energy load $L(d)$ which is defined as the expected power spent by a node at a distance $d$ from $O$. Note that the average power consumption level is equal for all nodes that are equidistant from the sink due to the inherent symmetry of the problem. An analytical expression for $L(d)$ is obtained by extending the geographical model presented in [9] and [5]. As shown in Fig. 1, for a node $n$ at distance $d$ from the center $O$, the average load taken by node $n$ is in proportion to $\left(S_{1}+S_{2}\right) / S_{2}$ where $S_{1}$ and $S_{2}$ can be approximated as follows.

$$
\begin{aligned}
& S_{1} \simeq \frac{\beta(d)}{2}\left(D^{2}-d^{2}\right), \quad S_{2} \simeq \frac{\pi}{2} t(d)^{2}, \quad d \geq t_{0} \\
& S_{1}=\pi D^{2}, \quad S_{2}=\pi t(d)^{2}, \quad d<t_{0}
\end{aligned}
$$

where $\beta(d)=2 \arcsin (t(d) / d)$. Then, the expected energy load of a node at distance $d$ is

$$
\begin{array}{rlrl}
L(d) & \simeq \frac{\left(S_{1}+S_{2}\right) \rho_{s} \lambda \varepsilon(d)}{S_{2}\left(\rho_{s}+\rho_{r}(d)\right)} & \\
& \simeq \frac{\left(\frac{\beta(d)}{2}\left(D^{2}-d^{2}\right)+\frac{\pi}{2} t^{2}(d)\right) \rho_{s} \lambda \varepsilon(d)}{\frac{\pi}{2} t^{2}(d) \rho(d)}, & & d \geq t_{0} \\
& =\frac{D^{2} \rho_{s} \lambda \varepsilon(d)}{t^{2}(d) \rho(d)}, & & d<t_{0}
\end{array}
$$

We observe the variation of expected load with respect to distance $d$ in Fig. 2 when there is no relay deployment or transmission power control (i.e., $\rho_{r}(d)=0, t(d)=t_{s}$ for all $0<d \leq D)$. The aforementioned energy bottleneck phenomenon can be clearly seen as the nodes in the proximity of the sink node deplete their energy rapidly and the sink node has no way to collect data any more even though a large part of the network is still "alive" [9]. Consequently, network lifetime is strictly limited by the energy depletion rate of these sensors that are close to the sink.

Following the discussion in [9], the expected network lifetime maximization problem can be reformulated as a energy load balancing problem. The corresponding optimization problem can be stated as follows:

$$
\min _{\rho(d), t(d)} \max _{0<d \leq D} L(d)
$$

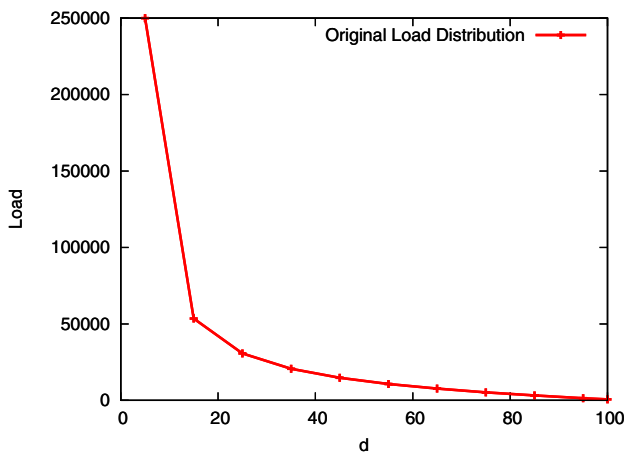

Fig. 2. Original load distribution without relay, $D=100, t_{s}=5, \lambda=1, a=4$, and $\rho_{s}=1 / \pi$

$$
\begin{gathered}
\text { s.t. } \mathrm{P}\left(\mathbf{G}(t(d), \mathcal{S} \cup \mathcal{R}) \in \mathbf{G}_{k}\right) \geq p_{0}, \\
t(d) \leq t_{s},
\end{gathered}
$$

\section{OPTIMAL RELAY DISTRIBUTION}

Due to shortest path routing assumption, the sensor nodes located at the outmost layer of the circle $C$ (i.e., sensor nodes that are at $D$ units away from $O$ ) forward only their locally generated data and do not relay any additional data generated by other sensor nodes (i.e., $L(D)=\lambda \varepsilon(D)$ ). $L(D)$ constitutes a lower bound on the load for all other sensors.

$$
L(d) \geq L(D)=\lambda \varepsilon(D), \quad \text { for all } 0<d \leq D .
$$

Note that deployment of additional relay nodes to the network has no effect on the lifetime of the nodes at distance $D$. Hence, for an initial battery energy level $B$, the expected network lifetime can be upper bounded by $\frac{B}{\lambda \varepsilon(D)}$ and it can only be achieved if (10) holds with equality, i.e.,

$$
L^{*}(d)=L(D), \text { for all } 0<d \leq D,
$$

where $L^{*}(d)$ represents the expected energy load at distance $d$ when relay nodes are optimally distributed to the network.

From (4) we can obtain the optimal relay distribution $\rho^{*}(d)$ in terms of the optimal transmission ranges $t^{*}(d)$

$$
\begin{aligned}
& \rho_{r}^{*}(d)=\frac{S_{1} \varepsilon(d)+S_{2}(\varepsilon(d)-\varepsilon(D))}{S_{2} \varepsilon(D)} \rho_{s} \\
& \rho_{r}^{*}(d)=\frac{S_{1}\left(t^{*}(d)\right)^{a}+S_{2}\left(\left(t^{*}(d)\right)^{a}-\left(t^{*}(D)\right)^{a}\right)}{S_{2}\left(t^{*}(D)\right)^{a}} \rho_{s} .
\end{aligned}
$$

Note that $t^{*}(d)$, the optimal transmission range to be used at a distance $d$ to $O$, is yet to be determined using the connectivity constraints.

It is possible to maintain such a load balance by merely deploying additional relay nodes to the network as will be shown in the following section. However, the number of relay nodes required can be reduced if relay deployment is coupled with transmission power control, i.e., by optimizing $t(d)$ for all $0<d \leq D$. In fact, the savings in required number of relays is rather significant which is presented in Section III-B. 


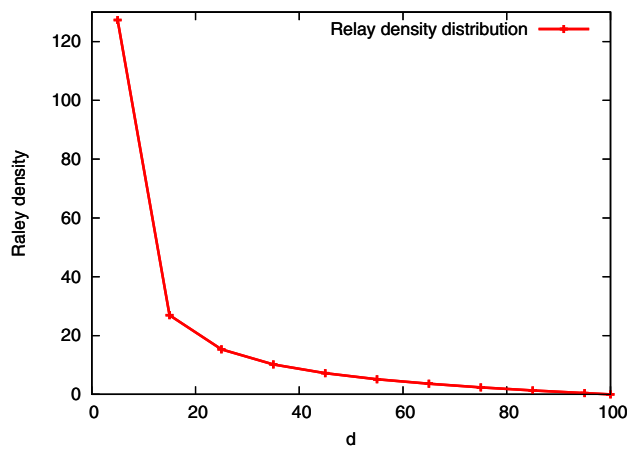

Fig. 3. Relay density, $D=100, t_{s}=5, \lambda=1$, a $=4$, and $\rho_{s}=1 / \pi$

\section{A. Optimal Relay Deployment with Homogeneous Transmis- sion Ranges}

In this section, we consider relay deployment under the condition that all sensors and relays have the same fixed transmission range, i.e., $t(d)=t_{s}$ for all $0<d \leq D$.

As there is no transmission power control involved with the optimization, the optimal relay distribution $\rho_{r}(d)$ can simply be obtained from (13) as

$$
\begin{array}{rlrl}
\rho_{r}(d) & = & \\
& =\frac{\beta(d)\left(D^{2}-d^{2}\right)}{\pi t_{s}{ }^{2}} \rho_{s} & & d>t_{s}, \\
& =\frac{D^{2}}{t_{s}{ }^{2}} \rho_{s} & & d \leq t_{s} .
\end{array}
$$

Fig. 3 shows the variation of relay density $\rho_{r}(d)$ with respect to $d$. We observe that a large amount of relays are needed to balance energy load. For instance, just in the the one-hop neighborhood of the sink node (an area of $\pi t_{s}^{2}$ ), the required number of relays is equal 10,000 , which is equivalent to the total number of sensors in the network. This is a rather large amount, even though the required relays decreases dramatically with increasing $d$. Such a high number of relays is required partly because the energy load of the sensors at the edge of the network $(L(D))$ is very low which causes a strict constraint for the sensor that are close to the sink. Moreover, here we did not exploit the potential benefits one can observe through transmission power control. In fact, in the following section we will show how the relay density can be reduced significantly by controlling the transmission ranges.

\section{B. Joint Relay Deployment and Power Control}

In the previous section we observed that a high relay node density is observed especially in the vicinity of the sink node. A high node density of wireless nodes can potentially incur severe interference. In this section, we will show that the relay density can be reduced efficiently by employing transmission power control.

It is clear that a shorter transmission range reduces the energy consumption of a node per unit data transmitted $(\varepsilon(d))$. As a result, the required number of relays to share the load can be reduced due to the reduction of expected load $(L(d))$ for such nodes. However, the transmission ranges cannot be reduced indefinitely. A certain level of connectivity should be maintained for the network to operate successfully. A straightforward treatment on the connectivity issue is to require the connectivity of the new network with the relays be no less than that of the original sensor network. This constitutes a constraint on the optimization problem as stated in (8).

In [3], it has been shown that a homogeneous ad hoc network can be modeled as a geometric random graph $\tilde{G}$ where the following inequalities hold:

$$
\begin{aligned}
& P\left(\tilde{G} \in G_{k}\right) \leq P\left(d_{\min } \geq k\right) \\
& P(\tilde{G} \text { is disconnected }) \geq 1-P\left(d_{\min }>0\right)
\end{aligned}
$$

Here $P\left(d_{\min } \geq k\right)$ is the probability that each node has at least $k$ neighbors, i.e., the network has a minimum degree $d_{\min } \geq k$. For the case of Poisson distributed $S$ nodes with a density $\rho_{s}$, transmission range $t_{s}$ this probability is equal to the following:

$$
P\left(d_{\min } \geq k\right)=\left(1-\sum_{n=0}^{k-1} \frac{\left(\rho_{s} \pi t_{s}^{2}\right)^{n}}{n !} e^{-\rho_{s} \pi t_{s}^{2}}\right)^{S}
$$

Moreover, it is shown in [3] that the bound given in (16) is tight when number of nodes is high. Therefore, it is reasonable to relate the probability of network connectivity to probability on the minimum degree of the network.

However, one should note that the connectivity results above are valid only under a homogeneous Poisson node distribution. Toward this end, we evenly divide the circle $C$ into a set of layers $\mathcal{M}=\{1, \ldots, M\}$, where the relay density in the $m^{\text {th }}$ layer is constant $\left(\rho_{m}\right)$. We also assume that all nodes in layer $m$ have the same transmission range $t_{m}$. The width of a layer is denoted as $\delta$ and is equal to $D / M .^{2}$ The distance from the $m^{\text {th }}$ layer to the sink position $O$ of $C, d_{m}$, represents the distance between $O$ and node $n$ that is located in layer $m$ and is equidistant from both boundaries of layer $m$. For simplicity we assume that $\rho_{M}=0$. Furthermore, it is convenient to divide the first layer into two parts as, i.e., $0<d \leq t_{0}$ and $t_{0}<d \leq \delta$ as $L(d)$ terms for these two segments are different according to (4). We will denote this inner layer as the layer $m=0$ in the following.

Since the uniform assumptions on the node density and transmission range within one layer still hold, we have the following for the overall network $\mathbf{G}(t(d), S \cup \mathcal{R})$ :

$$
\begin{aligned}
& P\left(\mathbf{G}(t(d), \mathcal{S} \cup \mathcal{R}) \in G_{k}\right) \leq P\left(d_{\min } \geq k\right) \\
& \simeq \prod_{m=1}^{M}\left(1-\sum_{n=0}^{k-1} \frac{\left(\left(\rho_{s}+\rho_{m}\right) \pi t_{m}^{2}\right)^{n}}{n !} e^{-\left(\rho_{s}+\rho_{m}\right) \pi t_{m}{ }^{2}}\right)^{\mathcal{S}_{m}+i R_{m}}
\end{aligned}
$$

where $S_{m}$ and $R_{m}$ denote the number of sensor and relay nodes located in the $m^{\text {th }}$ layer respectively.

Note that there is an inaccuracy involved in (19) due to neglecting the boundary effects between two adjacent layers where a node in one layer has its transmission range extend into the other layer. However, it is easy to see that this is

\footnotetext{
${ }^{2}$ The width $\delta$ is selected such that it is larger than $t_{s}$.
} 


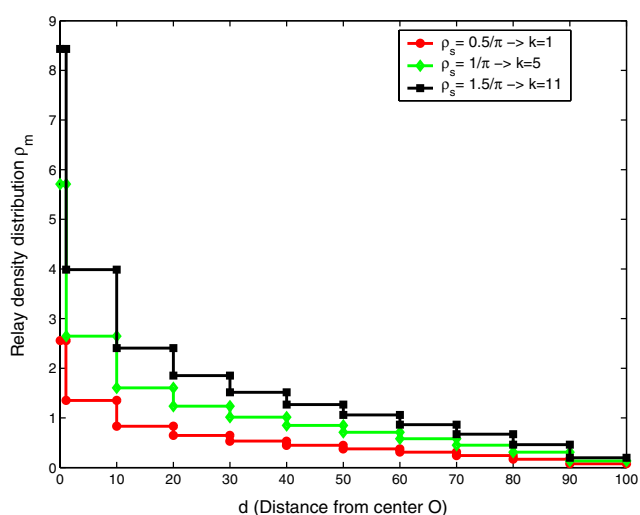

(a) Relay density distribution vs. $d$

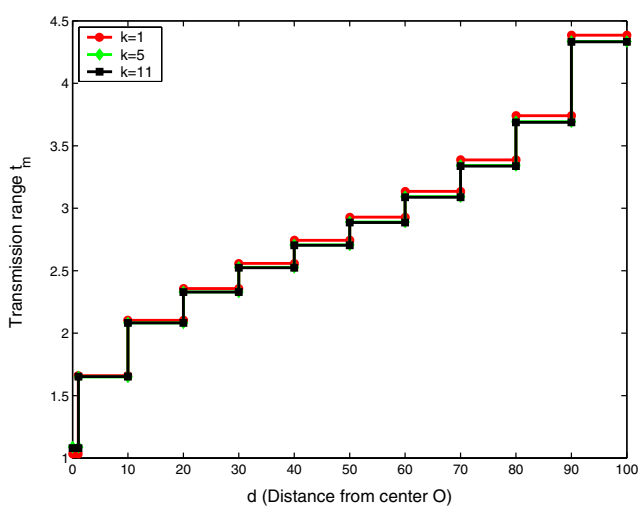

(b) Transmission range vs. $d$

Fig. 4. k-connectivity ( $\rho_{s}$ varies), $D=100, t_{s}=5, \lambda=1, a=3$, and $p_{0}=0.999$

rather a strict assumption due to the nature of data transport which is always along the direction from an outer layer to an inner layer. In fact, if a node in the $m^{\text {th }}$ layer has neighbor nodes at $(m-1)^{t h}$ layer where the node density is higher, it can only have more neighbors to send data than the case where the boundary effects are ignored. Therefore, the relay densities to be found using the above formulation will be an upper bound to the actual case.

With this simplifying assumption, for a given layer $m$ we can obtain the necessary relay density $\rho_{m}$ and the corresponding transmission range $t_{m}$ independent of other layer parameters by jointly satisfying (13) and (16) as follows:

$$
\begin{array}{rlr}
\rho_{m} & =\frac{D^{2} t_{m}{ }^{a}+t_{m}{ }^{2}\left(t_{m}{ }^{a}-t_{s}{ }^{a}\right)}{t_{m}{ }^{2} t_{s}{ }^{a}} \rho_{s}, & m=0 \\
& =\frac{\beta\left(d_{m}\right)\left(D^{2}-{d_{m}}^{2}\right) t_{m}{ }^{a}+\pi t_{m}{ }^{2}\left(t_{m}{ }^{a}-t_{s}{ }^{a}\right)}{\pi t_{m}{ }^{2} t_{s}{ }^{a}} \rho_{s}, & m>0
\end{array}
$$

and

$$
\begin{aligned}
P\left(d_{\text {min }}^{m}>k\right) & \geq p_{o} \\
\left(1-\sum_{n=0}^{k-1} \frac{\left(\left(\rho_{m}+\rho_{s}\right) \pi t_{m}^{2}\right)^{n}}{n !} e^{-\left(\rho_{m}+\rho_{s}\right) \pi t_{m}^{2}}\right)^{\mathcal{S}_{m} \cup \mathcal{R}_{m}} & \geq p_{o}
\end{aligned}
$$

where $d_{m i n}^{m}$ represents the minimum node degree in layer $m$.

The joint solution $\left(\rho_{m}, t_{m}\right)$ can be obtained from (20), (21) and (22) using an iterative algorithm. The necessary relay density and transmission ranges under various initial sensor density $\left(\rho_{s}\right)$ are depicted in Fig. 4. Note that for each $\rho_{s}$ we have a different network connectivity level. For instance, for $\rho_{s}=1 / \pi, P\left(\mathbf{G}\left(t_{s}, \mathcal{S}\right) \in \mathbf{G}_{5}\right)=0.999$. This changes the set of constraints of the optimization problem since the overall network is constrained to leave original connectivity conditions unchanged. The results demonstrate the tradeoffs between the relay density and transmission range with the distance $d$. By jointly considering the relay density distribution and the transmission range control (power control), we observe that much lower relay density can be achieved for the same expected network lifetime $B / L(D)$, comparing Fig. 4(a) with Fig. 3. Comparably, the number of relays required in the one-hop neighborhood of the sink node is 21 (relay density $\rho_{d}$ is equal to 5.71 when $\left.\rho_{s}=1 / \pi\right)$. Note that, under this solution not only the node density around the sink is lower, but also the transmission range in the vicinity of the sink is smaller as well $\left(t_{s}=5\right.$ vs. $\left.t_{0}=1.08\right)$. To be fair with the earlier analysis, we should also calculate the number of relays that are in the circle with an area of $\pi t_{s}{ }^{2}$. Using the values from Fig.4, the number of relays needed in the area of $\pi t_{s}^{2}$ is 219 (21 in the one-hop neighborhood and 198 nodes in the area between the circles with radius $d=1.08$ to $d=t_{s}=5$ ) compared to 10,000 when no transmission range control is used. Hence, the savings in the number of relays is significant making the proposed solution a feasible alternative for maximizing the network lifetime.

One question that may arise is the effect of the additional nodes on the effective throughput of the network. However, as the relay nodes do not generate additional traffic they do not constitute a degradation on the throughput capacity of the network. We consider the details of this issue in [11].

\section{Simulation RESUlts}

In this section, we present simulation results to verify the performance of the proposed relay deployment and transmission power control scheme. We compare performance of the proposed solution in Section III-B with the original sensor network, i.e., without addition of relay nodes and with a fixed transmission range $t_{s}$. Using the values for relay density and transmission ranges, our aim is to observe the effects of approximations made throughout the analysis. Every node is assigned an initial energy of $E=2000000$ units. We fix the related parameters the same as given in Fig. 4 with $k=5(k$ connectivity level) and the packet size is $\mu=0.5$.

We adapted the following neighbor-based load balancing routing protocol in the simulation. During the simulation, every node maintains a neighbor list that contains nodes within its transmission range that satisfy two conditions: (1) enough residual energy; (2) equal or smaller distance to the sink. When there is a packet, either generated locally or relayed from another node, it chooses the node in its neighbor list that has the maximum residual energy as its next-hop destination. Due to the broadcast nature of wireless communication, this information can be obtained when neighbours attach their residual energy value to the regular data or routing beacon packets. We assume a perfect MAC layer for simplicity. 


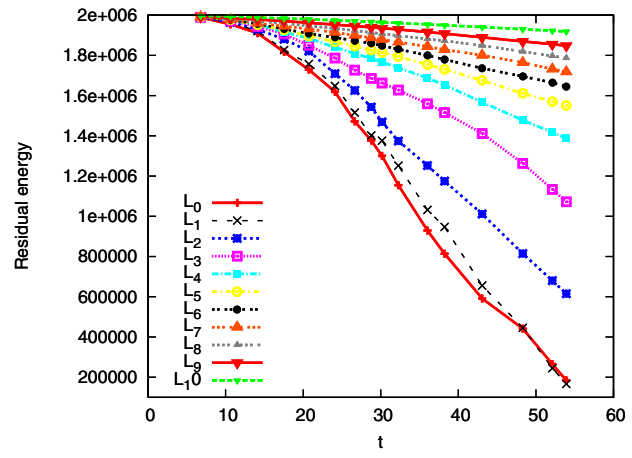

(a) Original sensor network without relays

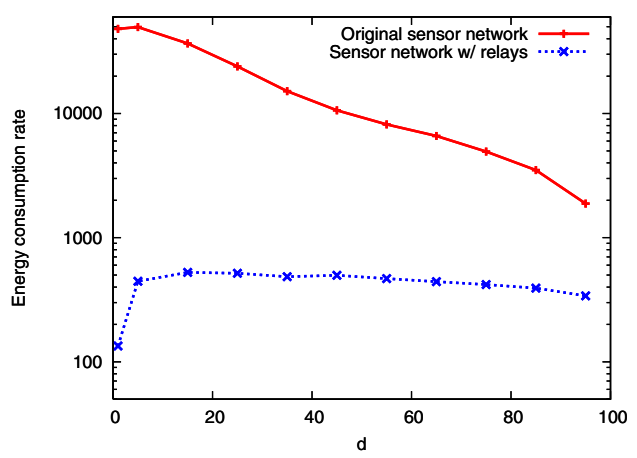

(b) Sensor network with relays

Fig. 5. Simulation result, $D=100, t_{s}=5, \lambda=1, a=4$, and $p_{0}=0.999$

The simulation results are depicted in Fig. 5(a) and Fig. 5(b). In Fig. 5(a), the $\mathrm{X}$-axis represents the actual time in the network and the Y-axis represents the average residual energy over all nodes within a layer. We observe that the simulation results confirm the analytical results, suggesting the errors due to approximations are negligible. For the original sensor network, the nodes in the inner layer deplete their energy very fast and the network dies roughly at time $t=64$. Fig. 5(b) shows the average energy consumption rate (per time unit) with respect to the distance $d$ for both original network and the one with relays and transmission range control. We observe that, in the later case, the energy of wireless nodes over all layers is consumed at a similar pace. Consequently, the lifetime of the network is prolonged dramatically after almost all nodes in the network deplete their energy. This is a very desirable result in terms of the scalability because we make the lifetime of the sensor network above 100 times longer than the original network by adding only about the same number of cheap relays as the number of original sensors and adjusting the transmission ranges.

\section{CONCLUSION}

Due to the multipoint-to-point communications pattern observed in general homogeneous sensor networks, the closer a sensor to the sink, the quicker it will deplete its battery. This unbalanced energy depletion phenomenon has become the bottleneck problem to elongate the lifetime of sensor networks.
In this paper, we propose to deploy wireless relay nodes in the sensor network as a simple method to solve this problem. These relay nodes are rather simple devices contrary to the common approach in the literature. This way a cost-effective solution to elongate the lifetime of sensor networks is proposed. By transforming the original homogeneous wireless sensor network to a heterogeneous sensor and relay hybrid network, the expected energy load is evenly distributed over all the wireless nodes (sensors and relays) so that all nodes will deplete batteries at the same time. We presented analytical results under different scenarios where the maximum transmission ranges can be either homogeneous or heterogeneous. In the heterogeneous transmission range case, we take the network connectivity into consideration. By jointly considering the load balance and network connectivity, the results showed that the maximum network lifetime could be achieved by adding relay nodes with relatively lower density, at the same time the maximum transmission ranges of both relay and sensor nodes can be significantly reduced. In this paper, we considered a common distribution for sensor nodes and exploit the properties to obtain the solution. However, the results can easily be extended under different sensor distribution models.

There are more problems that are worth further investigation. We have intentionally ignored the receiving and data processing energy consumption. In addition, we have considered only a single information sink that is located at the center of a circle that constitutes the network area and we made use of the inherent symmetry caused by these assumptions. Even though it is shown in [9] that locating the sink node at the center of a circular network is optimal in terms of energy efficient data collection in a static homogeneous sensor network, it is of interest to generalize the problem to the case where the sink node is not necessarily located at the center as well as to the case of multiple sinks.

\section{REFERENCES}

[1] I. F. Akyildiz, W. Su, Y. Sankarasubramaniam, and E. Cayirci. A survey on sensor networks. Computer Networks Journal, Vol. 38, no. 8, Aug. 2002

[2] M. Bhardwaj and A. P. Chandrakasan Bounding the lifetime of sensor networks via optimal role assingments. Proceedings of IEEE Infocom, 2002.

[3] C. Bettstetter. On the minimum node degree and connectivity of a wireless multihop network. Proceedings of IEEE MobiHoc, June 2002.

[4] B. Bollobás. Modern graph theory. Springer, 1998.

[5] Y. Ganjali and A. Keshavarzian. Load balancing in ad hoc networks: single-path routing vs. multi-path routing. Proceedings of IEEE Infocom, Mar. 2005.

[6] J. Gao and L. Zhang. Load balanced short path routing in wireless networks. Proceedings of IEEE Infocom, 2004.

[7] N. Li and J. Hou. Topology control in heterogeneous wireless networks: problems and solutions. Proceedings of IEEE Infocom, 2004.

[8] X. Liu and P. Mohapatra. On the deployment of wireless sensor networks. the Third International Workshop on Measurement, Modeling, and Performance Analysis of Wireless Sensor Networks (SenMetrics), 2005.

[9] J. Luo and J. P. Hubaux. Joint mobility and routing for lifetime elongation in wireless sensor networks. Proceedings of IEEE INFOCOM, 2005.

[10] S. M. Ross. Introduction to probability models. Academic Press, 2003.

[11] Y. Xin and T. Guven and M. A. Shayman. Limited relay deployment for sensor networks. In preparation. 